\title{
Erratum to: $C P$ Properties of Leptons within the Mirror Mechanism
}

\author{
I. T. Dyatlov* \\ Petersburg Nuclear Physics Institute, National Research Center Kurchatov Institute, Gatchina, 188300 Russia \\ Received May 18, 2021; revised May 18, 2021; accepted May 18, 2021
}

DOI: $10.1134 / \mathrm{S} 1063778821050173$

In the author's papers on the mirror mechanism, the properties of the lepton weak mixing matrix (PMNS matrix) are reproduced at zero Majorana parts of masses of light $\nu_{\mathrm{S} M}$ neutrinos. For heavy mirror neutrinos considered in the papers and produced by Majorana fermions whose masses have equal moduli, this approach is not correct. In this case, light $\nu_{\mathrm{S} M}$ include both the Dirac $\left(\mu_{\mathrm{D}}\right)$ and Majorana $\left(\mu_{M}\right)$ parts of the mass matrices (again, with equal mass moduli):

$$
\begin{gathered}
\left(\mu_{M}\right)_{a, b} \approx \sum_{n=0}^{2} A_{a n} \frac{\mu_{n}}{\left|M_{n}\right|^{2}} A_{n b}^{+} ; \\
\left(\mu_{M}\right)_{a b} \approx \pm \sum_{n=0}^{2} A_{a n} \frac{1}{M_{n}} A_{n b}^{T} ; \\
A \ll \mu \ll|M|, \quad a, b=1,2,3 .
\end{gathered}
$$

The notation is the same as in the above-mentioned papers. $M_{n}$ may be complex when the Majorana phases are present. $A$ is a real symmetric matrix.

Diagonalization of both parts of Eq. (1) (with $U U^{T}=1$ ) conserves all PMNS natural properties found in the papers under the same assumption on the inverse $\nu_{S M}$ spectrum. The reproduction stems from the general properties of the mirror symmetry model. But Dirac $\nu_{S M}$ neutrino mass allows now the lepton number breaking ( similarly to heavy neutrinos in the above-mentioned papers).

Other choices of the coefficients, e.g. various $\mathrm{Ma}-$ jorana masses and complex $A$, do not provide simple reproduction of the light neutrino mass spectra and of the properties of the PMNS matrix.

The original article can be found online at https://doi.org/10.1134/S1063778819020066

*E-mail: dyatlov@thd.pnpi.spb.ru 